\title{
Strong convergence theorems for quasi-nonexpansive mappings and maximal monotone operators in Hilbert spaces
}

\author{
Huan-chun Wu*, Cao-zong Cheng and De-ning Qu \\ Celebration of Professor SS Chang's 80th birthday
}

${ }^{*}$ Correspondence:

wuhuanchun@emails.bjut.edu.cn College of Applied Sciences, Beijing University of Technology, Beijing, 100124, China

\begin{abstract}
We present the strong convergence theorem for the iterative scheme for finding a common element of the fixed-point set of a quasi-nonexpansive mapping and the zero set of the sums of maximal monotone operators in Hilbert spaces. Our results extend and improve the recent results of Takahashi et al. (J. Optim. Theory Appl. 147:27-41, 2010) and Takahashi and Takahashi (Nonlinear Anal. 69:1025-1033, 2008). MSC: $47 \mathrm{H} 05 ; 47 \mathrm{H} 09 ; 47 \mathrm{~J} 25$
\end{abstract}

Keywords: quasi-nonexpansive mapping; maximal monotone operator; variational inequality

\section{Introduction}

Let $H$ be a real Hilbert space and let $K$ be a nonempty closed convex subset of $H$. Let $T: K \rightarrow K$ be a mapping. We denote by $\operatorname{Fix}(T)$ the fixed-point set of $T$, that is, $\operatorname{Fix}(T)=\{x \in K: T x=x\}$. A mapping $T: K \rightarrow K$ is nonexpansive if $\|T x-T y\| \leq\|x-y\|$ for all $x, y \in K$. Approximation methods for fixed points of nonexpansive mappings have attracted considerable attention (see [1-5]). A mapping $T: K \rightarrow K$ is quasi-nonexpansive if $\operatorname{Fix}(T) \neq \emptyset$ and $\|T x-y\| \leq\|x-y\|$ for all $x \in K$ and $y \in \operatorname{Fix}(T)$. It is well known that the fixed-point set of a quasi-nonexpansive mapping is closed and convex (see [6, 7]). There are some quasi-nonexpansive mappings which are not nonexpansive (see [8-10]). For example, the level set of a continuous convex function is characterized as the fixed-point set of a nonlinear mapping called the subgradient projection, which is not nonexpansive but quasi-nonexpansive. Quasi-nonexpansive mappings have been discussed in the recent literature (see [9-11]).

We say that a mapping $T: K \rightarrow K$ is demiclosed at zero if for any sequence $\left\{x_{n}\right\} \subset K$ which converges weakly to $x$, the strong convergence of the sequence $\left\{T x_{n}\right\}$ to zero implies $T x=0$. It is well known that $I-T$ is demiclosed whenever $T$ is nonexpansive. In fact, this property is satisfied for more general mappings (see [12, 13]).

Let $B$ be a mapping from $H$ into $2^{H}$. The effective domain of $B$ is denoted by $\operatorname{dom}(B)$, namely, $\operatorname{dom}(B)=\{x \in H: B x \neq \emptyset\}$. The graph of $B$ is

$$
\operatorname{Gra}(B)=\{(v, r) \in H \times H: r \in B v\} .
$$

○2014 Wu et al.; licensee Springer. This is an Open Access article distributed under the terms of the Creative Commons Attribution License (http://creativecommons.org/licenses/by/2.0), which permits unrestricted use, distribution, and reproduction in any medium, provided the original work is properly cited. 
A multi-valued mapping $B$ is said to be monotone if

$$
\langle x-y, u-v\rangle \geq 0 \quad \text { for all } x, y \in \operatorname{dom}(B), u \in B x \text {, and } v \in B y \text {. }
$$

A monotone operator $B$ is said to be maximal if its graph is not properly contained in the graph of any other monotone operator. For a maximal monotone operator $B$ on $H$ and $r>0$, we define a single-valued operator $J_{r B}=(I+r B)^{-1}: H \rightarrow \operatorname{dom}(B)$, which is called the resolvent of $B$ for $r$. It is well known that $J_{r B}$ is firmly nonexpansive, that is,

$$
\left\langle x-y, J_{r B} x-J_{r B} y\right\rangle \geq\left\|J_{r B} x-J_{r B} y\right\|^{2} \quad \text { for any } x, y \in H \text {. }
$$

A basic problem for maximal monotone operator $B$ is to

$$
\text { find } x \in H \quad \text { such that } \quad 0 \in B x \text {. }
$$

The classical method for solving problem (1.1) is the proximal point algorithm which was first introduced by Martinet [14]. Rockafellar [15] obtained the weak convergence of the proximal point algorithm for maximal monotone operators. Güler [16] constructed a proximal point iteration that converges weakly but not strongly. Some researchers have devoted their work to modifications of the proximal point algorithm in order to obtain the strong convergence theorem (see $[17,18]$ ). For a positive constant $\alpha$, a mapping $A: K \rightarrow H$ is said to be $\alpha$-inverse strongly monotone if

$$
\langle x-y, A x-A y\rangle \geq \alpha\|A x-A y\|^{2} \quad \text { for all } x, y \in K .
$$

We write $(A+B)^{-1} 0$ for the zero set of $A+B$, that is, $(A+B)^{-1} 0=\{x \in K: 0 \in(A+B) x\}$, where the mapping $A: C \rightarrow H$ is inverse strongly monotone and $B$ is maximal monotone. It is well known that $(A+B)^{-1} 0=\operatorname{Fix}\left(J_{\lambda B}(I-\lambda A)\right)$ for all $\lambda>0$ (see [19]). Takahashi et al. [20] presented the following iterative sequence. Let $u \in K, x_{1}=x \in K$ and let $\left\{x_{n}\right\}$ be a sequence generated by

$$
x_{n+1}=\alpha_{n} u+\left(1-\alpha_{n}\right) J_{\lambda_{n} B}\left(x_{n}-\lambda_{n} A x_{n}\right) .
$$

Under appropriate conditions they proved that the sequence $\left\{x_{n}\right\}$ converges strongly to a point $z_{0} \in(A+B)^{-1} 0$. Lin and Takahashi [21] introduced an iterative sequence that converges strongly to an element of $(A+B)^{-1} 0 \cap F^{-1} 0$, where $F$ is another maximal monotone operator. Takahashi et al. [22] established an iterative scheme for finding a point of $(A+B)^{-1} 0 \cap \operatorname{Fix}(T)$ as follows. Let $x_{1}=x \in K$ and let $\left\{x_{n}\right\}$ be a sequence generated by

$$
x_{n+1}=\beta_{n} x_{n}+\left(1-\beta_{n}\right) T\left[\alpha_{n} x+\left(1-\alpha_{n}\right) J_{\lambda_{n} B}\left(x_{n}-\lambda_{n} A x_{n}\right)\right] \text {, }
$$

where $T: K \rightarrow K$ is a nonexpansive mapping.

Motivated by the above results, especially by Chuang et al. [11] and Takahashi et al. [22], we obtain the strong convergence theorem for the iterative scheme for finding a common element of the fixed-point set of a quasi-nonexpansive mapping and the zero set of the sums of maximal monotone operators in Hilbert spaces. Our results extend and improve the recent results of [22] and [23]. 
The rest of this paper is organized as follows. Section 2 contains some important facts and tools. In Section 3, we introduce a new iterative scheme for finding a common element of the fixed-point set of a quasi-nonexpansive mapping and the zero set of the sums of maximal monotone operators, and we prove strong convergence theorem in Hilbert spaces.

\section{Preliminaries}

Throughout this paper, let $H$ be a real Hilbert space with inner product $\langle\cdot, \cdot\rangle$ and norm $\|\cdot\|$, and let $K$ be a nonempty closed convex subset of $H$. Let $\mathbb{N}$ be the set of positive integers. We denote the strong convergence and the weak convergence of $\left\{x_{n}\right\}$ to $x$ by $x_{n} \rightarrow x$ and $x_{n} \rightarrow x$, respectively. For any $x \in H$, there exists a unique point $P_{K} x \in K$ such that

$$
\left\|x-P_{K} x\right\| \leq\|x-y\|, \quad \forall y \in K
$$

$P_{K}$ is called the metric projection of $H$ onto $K$. Note that $P_{K}$ is a nonexpansive mapping. For $x \in H$ and $z \in K$, we have

$$
z=P_{K} x \quad \Longleftrightarrow \quad\langle x-z, y-z\rangle \leq 0 \quad \text { for every } y \in K
$$

Let $f$ be a proper lower semicontinuous convex function of $H$ into $(-\infty,+\infty]$. The subdifferential $\partial f$ of $f$ is defined as

$$
\partial f(x)=\{z \in H: f(y)-f(x) \geq\langle z, y-x\rangle, \forall y \in H\}
$$

for all $x \in H$. Rockafellar [24] claimed that $\partial f$ is a maximal monotone operator. Let $\delta_{K}$ be the indicator function of $K$, i.e.,

$$
\delta_{K}(x)= \begin{cases}0, & x \in K, \\ +\infty, & x \notin K .\end{cases}
$$

The subdifferential $\partial \delta_{K}$ of $\delta_{K}$ is a maximal monotone operator since $\delta_{K}$ is a proper lower semicontinuous convex function on $H$. The resolvent $J_{r \partial \delta_{K}}$ of $\partial \delta_{K}$ for $r$ is $P_{K}$ (see [21]).

Let $A: K \rightarrow H$ be a nonlinear mapping. The variational inequality problem is to find $\bar{x} \in K$ such that

$$
\langle A \bar{x}, y-\bar{x}\rangle \geq 0 \quad \text { for every } y \in K \text {. }
$$

The solution set of $(2.3)$ is denoted by $V I(K, A)$. Some methods have been proposed to study the variational inequality problem (see [25-28] and the references therein). It is easy to see that $\operatorname{VI}(K, A)=\left(A+\partial \delta_{K}\right)^{-1} 0$, where $A$ is an inverse strongly monotone mapping of $K$ into $H$ (for more details, see [21]).

We collect some useful lemmas.

Lemma 2.1 [29] Let $A: K \rightarrow H$ be an $\alpha$-inverse strongly monotone mapping. For all $x, y \in$ $K$ and $\lambda>0$, we have

$$
\|(I-\lambda A) x-(I-\lambda A) y\|^{2} \leq\|x-y\|^{2}+\lambda(\lambda-2 \alpha)\|A x-A y\|^{2} .
$$

In particular, if $0<\lambda \leq 2 \alpha$, then $I-\lambda A$ is a nonexpansive mapping. 
Lemma 2.2 [30] Let $\left\{\Gamma_{n}\right\}$ be a sequence of real numbers that does not decrease at infinity in the sense that there exists a subsequence $\left\{\Gamma_{n_{j}}\right\}$ of $\left\{\Gamma_{n}\right\}$ such that

$$
\Gamma_{n_{j}}<\Gamma_{n_{j}+1} \text { for all } j \in \mathbb{N} \text {. }
$$

Define the sequence $\{\tau(n)\}_{n \geq n_{0}}$ of integers as follows:

$$
\tau(n)=\max _{k}\left\{k \leq n: \Gamma_{k}<\Gamma_{k+1}\right\},
$$

where $n_{0} \in \mathbb{N}$ such that $\left\{k \leq n_{0}: \Gamma_{k}<\Gamma_{k+1}\right\} \neq \emptyset$. Then, for all $n \geq n_{0}$, the following hold:

(1) $\tau(n) \leq \tau(n+1) \leq \cdots$ and $\tau(n) \rightarrow \infty$;

(2) $\Gamma_{\tau(n)} \leq \Gamma_{\tau(n)+1}$ and $\Gamma_{n} \leq \Gamma_{\tau(n)+1}$.

Lemma 2.3 [22] Let B be a maximal monotone operator on $H$. Then the following holds:

$$
\frac{s-t}{s}\left\langle J_{s B} x-J_{t B} x, J_{s B} x-x\right\rangle \geq\left\|J_{s B} x-J_{t B} x\right\|^{2}
$$

for all $s, t>0$ and $x \in H$.

The following lemma is an immediate consequence of the inner product on $H$.

Lemma 2.4 For all $x, y \in H$, the inequality $\|x+y\|^{2} \leq\|x\|^{2}+2\langle y, x+y\rangle$ holds.

Lemma 2.5 [31] Let $\left\{a_{n}\right\}$ be a sequence of nonnegative real numbers satisfying $a_{n+1} \leq(1-$ $\left.\alpha_{n}\right) a_{n}+\alpha_{n} \beta_{n}$, where

(i) $\left\{\alpha_{n}\right\} \subset(0,1), \sum_{n=1}^{\infty} \alpha_{n}=\infty$;

(ii) $\lim \sup _{n \rightarrow \infty} \beta_{n} \leq 0$.

Then $\lim _{n \rightarrow \infty} a_{n}=0$.

\section{Strong convergence theorems}

In this section, a new iterative scheme for finding a common element of the fixed-point set of a quasi-nonexpansive mapping and the zero set of the sums of maximal monotone operators is presented.

Theorem 3.1 Let $K$ be a nonempty closed convex subset of a real Hilbert space $H$. Let A : $K \rightarrow H$ and $C: K \rightarrow H$ be $\alpha$-inverse strongly monotone and $\gamma$-inverse strongly monotone, respectively. Suppose that $B$ and $D$ are maximal monotone operators on $H$ such that the domains of $B$ and $D$ are contained in $K$ and that $T: K \rightarrow K$ is a quasi-nonexpansive mapping such that $I-T$ is demiclosed at zero. Assume that $\Omega:=\operatorname{Fix}(T) \cap(A+B)^{-1} 0 \cap(C+D)^{-1} 0 \neq \emptyset$. Let $\left\{\alpha_{n}\right\}$ and $\left\{\beta_{n}\right\}$ be sequences in $(0,1)$ and let $\left\{u_{n}\right\}$ be a sequence in $K$. Let $\left\{x_{n}\right\}$ be a sequence generated by

$$
\left\{\begin{array}{l}
x_{1} \in K \quad \text { chosen arbitrarily, } \\
y_{n}=\alpha_{n} u_{n}+\left(1-\alpha_{n}\right) J_{\lambda_{n} B}\left(x_{n}-\lambda_{n} A x_{n}\right), \\
z_{n}=J_{\psi_{n} D}\left(y_{n}-\psi_{n} C y_{n}\right), \\
x_{n+1}=\beta_{n} x_{n}+\left(1-\beta_{n}\right) T z_{n} .
\end{array}\right.
$$


Suppose the following conditions are satisfied:

(c1) $\lim _{n \rightarrow \infty} \alpha_{n}=0$ and $\sum_{n=1}^{\infty} \alpha_{n}=\infty$;

(c2) $\lim _{n \rightarrow \infty} u_{n}=u$;

(c3) $0<\liminf _{n \rightarrow \infty} \beta_{n} \leq \limsup _{n \rightarrow \infty} \beta_{n}<1$;

(c4) $0<a \leq \lambda_{n} \leq b<2 \alpha$;

(c5) $0<c \leq \psi_{n} \leq d<2 \gamma$.

Then the sequence $\left\{x_{n}\right\}$ converges strongly to $P_{\Omega} u$.

Proof Observe that the set $\Omega$ is closed and convex since Fix $(T),(A+B)^{-1} 0$ and $(C+D)^{-1} 0$ are closed and convex.

By Lemma 2.1, for any $p \in \Omega$, we have

$$
\left\|z_{n}-p\right\|=\left\|J_{\psi_{n} D}\left(y_{n}-\psi_{n} C y_{n}\right)-J_{\psi_{n} D}\left(p-\psi_{n} C p\right)\right\| \leq\left\|y_{n}-p\right\|
$$

and

$$
\begin{aligned}
\left\|y_{n}-p\right\| & \leq \alpha_{n}\left\|u_{n}-p\right\|+\left(1-\alpha_{n}\right)\left\|J_{\lambda_{n} B}\left(I-\lambda_{n} A\right) x_{n}-p\right\| \\
& \leq \alpha_{n}\left\|u_{n}-p\right\|+\left(1-\alpha_{n}\right)\left\|x_{n}-p\right\| .
\end{aligned}
$$

It follows that

$$
\begin{aligned}
\left\|x_{n+1}-p\right\| & \leq \beta_{n}\left\|x_{n}-p\right\|+\left(1-\beta_{n}\right)\left\|z_{n}-p\right\| \\
& \leq\left[1-\alpha_{n}\left(1-\beta_{n}\right)\right]\left\|x_{n}-p\right\|+\alpha_{n}\left(1-\beta_{n}\right)\left\|u_{n}-p\right\| \\
& \leq \max \left\{\left\|x_{n}-p\right\|,\left\|u_{n}-p\right\|\right\} .
\end{aligned}
$$

The sequence $\left\{u_{n}\right\}$ is bounded due to condition (c2). Hence there exists a positive number $L$ such that $\sup _{n}\left\{\left\|u_{n}-p\right\|\right\} \leq L$. By a simple inductive process, we have

$$
\left\|x_{n+1}-p\right\| \leq \max \left\{\left\|x_{1}-p\right\|, L\right\},
$$

which shows that $\left\{x_{n}\right\}$ is bounded. So are $\left\{y_{n}\right\}$ and $\left\{z_{n}\right\}$.

Note that

$$
2\left\langle x_{n+1}-x_{n}, x_{n}-p\right\rangle=\left\|x_{n+1}-p\right\|^{2}-\left\|x_{n}-p\right\|^{2}-\left\|x_{n+1}-x_{n}\right\|^{2}
$$

and

$$
x_{n+1}-x_{n}=\left(1-\beta_{n}\right)\left(T z_{n}-x_{n}\right) .
$$

Thus we get

$$
\begin{aligned}
& \left\|x_{n+1}-p\right\|^{2}-\left\|x_{n}-p\right\|^{2}-\left\|x_{n+1}-x_{n}\right\|^{2} \\
& \quad=2\left\langle x_{n+1}-x_{n}, x_{n}-p\right\rangle \\
& \quad=\left(1-\beta_{n}\right)\left[\left\|T z_{n}-p\right\|^{2}-\left\|T z_{n}-x_{n}\right\|^{2}-\left\|x_{n}-p\right\|^{2}\right]
\end{aligned}
$$




$$
\begin{aligned}
& \leq\left(1-\beta_{n}\right)\left[\left\|z_{n}-p\right\|^{2}-\left\|T z_{n}-x_{n}\right\|^{2}-\left\|x_{n}-p\right\|^{2}\right] \\
& \leq \alpha_{n}\left(1-\beta_{n}\right)\left\|u_{n}-p\right\|^{2}-\left(1-\beta_{n}\right)\left\|T z_{n}-x_{n}\right\|^{2}
\end{aligned}
$$

and

$$
\begin{aligned}
& \left\|x_{n+1}-p\right\|^{2}-\left\|x_{n}-p\right\|^{2}-\left(1-\beta_{n}\right)^{2}\left\|T z_{n}-x_{n}\right\|^{2} \\
& \quad \leq \alpha_{n}\left(1-\beta_{n}\right)\left\|u_{n}-p\right\|^{2}-\left(1-\beta_{n}\right)\left\|T z_{n}-x_{n}\right\|^{2} .
\end{aligned}
$$

This implies that

$$
\left(1-\beta_{n}\right) \beta_{n}\left\|T z_{n}-x_{n}\right\|^{2} \leq\left\|x_{n}-p\right\|^{2}-\left\|x_{n+1}-p\right\|^{2}+\alpha_{n}\left(1-\beta_{n}\right)\left\|u_{n}-p\right\|^{2} .
$$

Set $\Gamma_{n}=\left\|x_{n}-p_{0}\right\|^{2}$, where $p_{0}=P_{\Omega} u$. We divide the rest proof into two cases.

Case 1. Suppose that $\Gamma_{n+1} \leq \Gamma_{n}$ for all $n \in \mathbb{N}$. In this case, the $\operatorname{limit}_{\lim _{n \rightarrow \infty}} \Gamma_{n}$ exists and then $\lim _{n \rightarrow \infty}\left(\Gamma_{n+1}-\Gamma_{n}\right)=0$. We obtain

$$
\lim _{n \rightarrow \infty}\left\|T z_{n}-x_{n}\right\|=0
$$

which implies

$$
\lim _{n \rightarrow \infty}\left\|x_{n+1}-x_{n}\right\|=\lim _{n \rightarrow \infty}\left(1-\beta_{n}\right)\left\|T z_{n}-x_{n}\right\|=0 .
$$

Note that

$$
\begin{aligned}
& \left\|J_{\lambda_{n} B}\left(x_{n}-\lambda_{n} A x_{n}\right)-p_{0}\right\|^{2} \\
& \quad \leq\left\|\left(I-\lambda_{n} A\right) x_{n}-\left(I-\lambda_{n} A\right) p_{0}\right\|^{2} \\
& \quad \leq\left\|x_{n}-p_{0}\right\|^{2}+\lambda_{n}\left(\lambda_{n}-2 \alpha\right)\left\|A x_{n}-A p_{0}\right\|^{2} .
\end{aligned}
$$

It follows that

$$
\begin{aligned}
&\left\|x_{n+1}-p_{0}\right\|^{2} \\
& \leq \beta_{n}\left\|x_{n}-p_{0}\right\|^{2}+\left(1-\beta_{n}\right)\left[\alpha_{n}\left\|u_{n}-p_{0}\right\|^{2}+\left(1-\alpha_{n}\right)\left\|J_{\lambda_{n} B}\left(x_{n}-\lambda_{n} A x_{n}\right)-p_{0}\right\|^{2}\right] \\
& \leq \beta_{n}\left\|x_{n}-p_{0}\right\|^{2}+\alpha_{n}\left(1-\beta_{n}\right)\left\|u_{n}-p_{0}\right\|^{2} \\
&+\left(1-\alpha_{n}\right)\left(1-\beta_{n}\right)\left[\left\|x_{n}-p_{0}\right\|^{2}+\lambda_{n}\left(\lambda_{n}-2 \alpha\right)\left\|A x_{n}-A p_{0}\right\|^{2}\right] \\
& \leq\left\|x_{n}-p_{0}\right\|^{2}+\alpha_{n}\left(1-\beta_{n}\right)\left\|u_{n}-p_{0}\right\|^{2} \\
&+\left(1-\alpha_{n}\right)\left(1-\beta_{n}\right) \lambda_{n}\left(\lambda_{n}-2 \alpha\right)\left\|A x_{n}-A p_{0}\right\|^{2},
\end{aligned}
$$

which yields

$$
\begin{aligned}
& \left(1-\alpha_{n}\right)\left(1-\beta_{n}\right) \lambda_{n}\left(2 \alpha-\lambda_{n}\right)\left\|A x_{n}-A p_{0}\right\|^{2} \\
& \quad \leq\left\|x_{n}-p_{0}\right\|^{2}-\left\|x_{n+1}-p_{0}\right\|^{2}+\alpha_{n}\left(1-\beta_{n}\right)\left\|u_{n}-p_{0}\right\|^{2} .
\end{aligned}
$$


Therefore we get

$$
\lim _{n \rightarrow \infty}\left\|A x_{n}-A p_{0}\right\|=0
$$

In a similar way, we have

$$
\lim _{n \rightarrow \infty}\left\|C y_{n}-C p_{0}\right\|=0
$$

Letting $h_{n}=J_{\lambda_{n} B}\left(I-\lambda_{n} A\right) x_{n}$, we have

$$
\begin{aligned}
\left\|h_{n}-p_{0}\right\|^{2} & =\left\|J_{\lambda_{n} B}\left(I-\lambda_{n} A\right) x_{n}-J_{\lambda_{n} B}\left(I-\lambda_{n} A\right) p_{0}\right\|^{2} \\
& \leq\left\langle\left(I-\lambda_{n} A\right) x_{n}-\left(I-\lambda_{n} A\right) p_{0}, h_{n}-p_{0}\right\rangle \\
& \leq \frac{1}{2}\left[\left\|x_{n}-p_{0}\right\|^{2}+\left\|h_{n}-p_{0}\right\|^{2}-\left\|\left(x_{n}-h_{n}\right)-\lambda_{n}\left(A x_{n}-A p_{0}\right)\right\|^{2}\right],
\end{aligned}
$$

from which one deduces that

$$
\left\|h_{n}-p_{0}\right\|^{2} \leq\left\|x_{n}-p_{0}\right\|^{2}-\left\|\left(x_{n}-h_{n}\right)-\lambda_{n}\left(A x_{n}-A p_{0}\right)\right\|^{2} .
$$

Using (3.1), we see that

$$
\begin{aligned}
&\left\|x_{n+1}-p_{0}\right\|^{2} \\
& \leq \beta_{n}\left\|x_{n}-p_{0}\right\|^{2}+\left(1-\beta_{n}\right)\left[\alpha_{n}\left\|u_{n}-p_{0}\right\|^{2}+\left(1-\alpha_{n}\right)\left\|h_{n}-p_{0}\right\|^{2}\right] \\
& \leq \beta_{n}\left\|x_{n}-p_{0}\right\|^{2}+\alpha_{n}\left(1-\beta_{n}\right)\left\|u_{n}-p_{0}\right\|^{2} \\
& \quad+\left(1-\alpha_{n}\right)\left(1-\beta_{n}\right)\left(\left\|x_{n}-p_{0}\right\|^{2}-\left\|x_{n}-h_{n}\right\|^{2}+2 \lambda_{n}\left\langle A x_{n}-A p_{0}, x_{n}-h_{n}\right\rangle\right) \\
& \leq\left\|x_{n}-p_{0}\right\|^{2}+\alpha_{n}\left(1-\beta_{n}\right)\left\|u_{n}-p_{0}\right\|^{2} \\
& \quad-\left(1-\alpha_{n}\right)\left(1-\beta_{n}\right)\left\|x_{n}-h_{n}\right\|^{2}+2\left(1-\alpha_{n}\right)\left(1-\beta_{n}\right) \lambda_{n}\left\langle A x_{n}-A p_{0}, x_{n}-h_{n}\right\rangle .
\end{aligned}
$$

Thus,

$$
\begin{aligned}
& \left(1-\alpha_{n}\right)\left(1-\beta_{n}\right)\left\|x_{n}-h_{n}\right\|^{2} \\
& \leq \quad\left\|x_{n}-p_{0}\right\|^{2}-\left\|x_{n+1}-p_{0}\right\|^{2}+\alpha_{n}\left(1-\beta_{n}\right)\left\|u_{n}-p_{0}\right\|^{2} \\
& \quad+2\left(1-\alpha_{n}\right)\left(1-\beta_{n}\right) \lambda_{n}\left\langle A x_{n}-A p_{0}, x_{n}-h_{n}\right\rangle .
\end{aligned}
$$

It follows from (3.5) that

$$
\lim _{n \rightarrow \infty}\left\|x_{n}-h_{n}\right\|=0 \text {. }
$$

This implies that

$$
\lim _{n \rightarrow \infty}\left\|x_{n}-y_{n}\right\|=0
$$

By (3.6), a similar argument shows that

$$
\lim _{n \rightarrow \infty}\left\|y_{n}-z_{n}\right\|=0
$$




$$
\left\|T z_{n}-z_{n}\right\| \leq\left\|T z_{n}-x_{n}\right\|+\left\|x_{n}-y_{n}\right\|+\left\|y_{n}-z_{n}\right\|,
$$

combining (3.3), (3.8) and (3.9) gives

$$
\lim _{n \rightarrow \infty}\left\|T z_{n}-z_{n}\right\|=0
$$

Next we prove that

$$
\limsup _{n \rightarrow \infty}\left\langle u-p_{0}, y_{n}-p_{0}\right\rangle \leq 0
$$

To show this inequality, we choose a subsequence $\left\{y_{n_{j}}\right\}$ of $\left\{y_{n}\right\}$ such that

$$
\limsup _{n \rightarrow \infty}\left\langle u-p_{0}, y_{n}-p_{0}\right\rangle=\lim _{j \rightarrow \infty}\left\langle u-p_{0}, y_{n_{j}}-p_{0}\right\rangle
$$

In view of the boundedness of $\left\{y_{n_{j}}\right\}$, without loss of generality, we assume that $y_{n_{j}} \rightarrow \omega$. Now we show that $\omega \in \Omega$. According to the fact that $\left\{y_{n}\right\}$ is contained in $K$ and $K$ is a closed convex set, one has $\omega \in \Omega$.

Note that the expressions (3.8) and (3.9) yield $x_{n_{j}} \rightarrow \omega$ and $z_{n_{j}} \rightarrow \omega$. By the fact that $I-T$ is demiclosed at zero, the expression (3.10) implies $\omega \in \operatorname{Fix}(T)$.

We prove that $\omega \in(A+B)^{-1} 0$. Due to (c4), there is a subsequence $\left\{\lambda_{n_{j_{i}}}\right\}$ of $\left\{\lambda_{n_{j}}\right\}$ such that $\lambda_{n_{j_{i}}} \rightarrow \lambda_{0} \in[a, b]$. Without loss of generality, we assume that $\lambda_{n_{j}} \rightarrow \lambda_{0}$. Observe that

$$
\begin{aligned}
\| x_{n_{j}}- & J_{\lambda_{0} B}\left(I-\lambda_{0} A\right) x_{n_{j}} \| \\
\leq & \left\|x_{n_{j}}-y_{n_{j}}\right\|+\left\|y_{n_{j}}-\left[\alpha_{n_{j}} u_{n_{j}}+\left(1-\alpha_{n_{j}}\right) J_{\lambda_{0} B}\left(I-\lambda_{0} A\right) x_{n_{j}}\right]\right\| \\
& +\left\|\alpha_{n_{j}} u_{n_{j}}+\left(1-\alpha_{n_{j}}\right) J_{\lambda_{0} B}\left(I-\lambda_{0} A\right) x_{n_{j}}-J_{\lambda_{0} B}\left(I-\lambda_{0} A\right) x_{n_{j}}\right\| \\
\leq & \left\|x_{n_{j}}-y_{n_{j}}\right\|+\left(1-\alpha_{n_{j}}\right)\left\|J_{\lambda_{n_{j}} B}\left(I-\lambda_{n_{j}} A\right) x_{n_{j}}-J_{\lambda_{0} B}\left(I-\lambda_{0} A\right) x_{n_{j}}\right\| \\
& +\alpha_{n_{j}}\left\|u_{n_{j}}-J_{\lambda_{0} B}\left(I-\lambda_{0} A\right) x_{n_{j}}\right\| \\
\leq & \left\|x_{n_{j}}-y_{n_{j}}\right\|+\left(1-\alpha_{n_{j}}\right)\left[\left\|J_{\lambda_{n_{j}} B}\left(I-\lambda_{n_{j}} A\right) x_{n_{j}}-J_{\lambda_{0} B}\left(I-\lambda_{n_{j}} A\right) x_{n_{j}}\right\|\right. \\
& \left.+\left\|J_{\lambda_{0} B}\left(I-\lambda_{n_{j}} A\right) x_{n_{j}}-J_{\lambda_{0} B}\left(I-\lambda_{0} A\right) x_{n_{j}}\right\|\right]+\alpha_{n_{j}}\left\|u_{n_{j}}-J_{\lambda_{0} B}\left(I-\lambda_{0} A\right) x_{n_{j}}\right\| \\
\leq & \left\|x_{n_{j}}-y_{n_{j}}\right\|+\left(1-\alpha_{n_{j}}\right)\left[\frac{\left|\lambda_{0}-\lambda_{n_{j}}\right|}{\lambda_{0}}\left\|J_{\lambda_{0} B}\left(I-\lambda_{n_{j}} A\right) x_{n_{j}}-\left(I-\lambda_{n_{j}} A\right) x_{n_{j}}\right\|\right. \\
& \left.+\left|\lambda_{n_{j}}-\lambda_{0}\right|\left\|A x_{n_{j}}\right\|\right]+\alpha_{n_{j}}\left\|u_{n_{j}}-J_{\lambda_{0} B}\left(I-\lambda_{0} A\right) x_{n_{j}}\right\| .
\end{aligned}
$$

Hence,

$$
\lim _{j \rightarrow \infty}\left\|x_{n_{j}}-J_{\lambda_{0} B}\left(I-\lambda_{0} A\right) x_{n_{j}}\right\|=0
$$

Since $J_{\lambda_{0} B}\left(I-\lambda_{0} A\right)$ is nonexpansive, the demiclosedness for a nonexpansive mapping implies that $\omega \in \operatorname{Fix}\left(J_{\lambda_{0} B}\left(I-\lambda_{0} A\right)\right)$, that is, $\omega \in(A+B)^{-1} 0$. 
Note that

$$
\begin{aligned}
\| y_{n_{i}} & -J_{\psi_{0} D}\left(I-\psi_{0} C\right) y_{n_{i}} \| \\
\leq & \left\|y_{n_{i}}-z_{n_{i}}\right\|+\left\|J_{\psi_{n_{i}} D}\left(I-\psi_{n_{i}} C\right) y_{n_{i}}-J_{\psi_{0} D}\left(I-\psi_{0} C\right) y_{n_{i}}\right\| \\
\leq & \left\|y_{n_{i}}-z_{n_{i}}\right\|+\left\|J_{\psi_{n_{i}} D}\left(I-\psi_{n_{i}} C\right) y_{n_{i}}-J_{\psi_{n_{i}} D}\left(I-\psi_{0} C\right) y_{n_{i}}\right\| \\
& +\left\|J_{\psi_{n_{i}} D}\left(I-\psi_{0} C\right) y_{n_{i}}-J_{\psi_{0} D}\left(I-\psi_{0} C\right) y_{n_{i}}\right\| .
\end{aligned}
$$

Using a similar argument, we get $\omega \in(C+D)^{-1} 0$. In fact, we have obtained $\omega \in \Omega$.

By (3.12) and (2.1), we have

$$
\begin{aligned}
\limsup _{n \rightarrow \infty}\left\langle u-p_{0}, y_{n}-p_{0}\right\rangle & =\lim _{j \rightarrow \infty}\left\langle u-p_{0}, y_{n_{j}}-p_{0}\right\rangle \\
& =\left\langle u-p_{0}, \omega-p_{0}\right\rangle \\
& \leq 0 .
\end{aligned}
$$

The inequality (3.11) is obtained.

Finally, we prove that $x_{n} \rightarrow p_{0}$. With the help of Lemma 2.4, we obtain

$$
\begin{aligned}
& \left\|x_{n+1}-p_{0}\right\|^{2} \\
& \quad \leq \beta_{n}\left\|x_{n}-p_{0}\right\|^{2}+\left(1-\beta_{n}\right)\left\|y_{n}-p_{0}\right\|^{2} \\
& \quad \leq \beta_{n}\left\|x_{n}-p_{0}\right\|^{2}+\left(1-\beta_{n}\right)\left[\left(1-\alpha_{n}\right)\left\|x_{n}-p_{0}\right\|^{2}+2 \alpha_{n}\left\langle u_{n}-p_{0}, y_{n}-p_{0}\right\rangle\right] \\
& \quad \leq\left[1-\alpha_{n}\left(1-\beta_{n}\right)\right]\left\|x_{n}-p_{0}\right\|^{2}+2 \alpha_{n}\left(1-\beta_{n}\right)\left(\left\langle u_{n}-u, y_{n}-p_{0}\right\rangle+\left\langle u-p_{0}, y_{n}-p_{0}\right\rangle\right) .
\end{aligned}
$$

It follows from (3.11) and Lemma 2.5 that $\left\{x_{n}\right\}$ converges strongly to $p_{0}$.

Case 2. Suppose that there exists a subsequence $\left\{\Gamma_{n_{i}}\right\}$ of $\left\{\Gamma_{n}\right\}$ such that

$$
\Gamma_{n_{i}}<\Gamma_{n_{i}+1} \quad \text { for all } i \in \mathbb{N} \text {. }
$$

We define $\tau: \mathbb{N} \rightarrow \mathbb{N}$ by

$$
\tau(n)=\max \left\{k \leq n: \Gamma_{k}<\Gamma_{k+1}\right\} .
$$

Lemma 2.2 shows that $\Gamma_{\tau(n)} \leq \Gamma_{\tau(n)+1}$. Therefore we have from (3.2)

$$
\lim _{n \rightarrow \infty}\left\|T z_{\tau(n)}-x_{\tau(n)}\right\|=0
$$

and

$$
\lim _{n \rightarrow \infty}\left\|x_{\tau(n)+1}-x_{\tau(n)}\right\|=0 .
$$

As in the proof of Case 1, we obtain

$$
\limsup _{n \rightarrow \infty}\left\langle u-p_{0}, y_{\tau(n)}-p_{0}\right\rangle \leq 0 .
$$


Observe that

$$
\begin{aligned}
& \left\|x_{\tau(n)+1}-p_{0}\right\|^{2} \\
& \leq\left[1-\alpha_{\tau(n)}\left(1-\beta_{\tau(n)}\right)\right]\left\|x_{\tau(n)}-p_{0}\right\|^{2} \\
& \quad+2 \alpha_{\tau(n)}\left(1-\beta_{\tau(n)}\right)\left(\left\langle u_{\tau(n)}-u, y_{\tau(n)}-p_{0}\right\rangle+\left\langle u-p_{0}, y_{\tau(n)}-p_{0}\right\rangle\right) .
\end{aligned}
$$

It follows that

$$
\left\|x_{\tau(n)}-p_{0}\right\|^{2} \leq 2\left(\left\langle u_{\tau(n)}-u, y_{\tau(n)}-p_{0}\right\rangle+\left\langle u-p_{0}, y_{\tau(n)}-p_{0}\right\rangle\right)
$$

which implies that

$$
\limsup _{n \rightarrow \infty}\left\|x_{\tau(n)}-p_{0}\right\|^{2} \leq 0
$$

Thus we get

$$
\lim _{n \rightarrow \infty}\left\|x_{\tau(n)}-p_{0}\right\|=0
$$

It follows from (3.15) and (3.18) that

$$
\lim _{n \rightarrow \infty}\left\|x_{\tau(n)+1}-p_{0}\right\|=0
$$

Lemma 2.2 implies that

$$
\lim _{n \rightarrow \infty}\left\|x_{n}-p_{0}\right\|=0
$$

The proof is completed.

The following result is a direct consequence of Theorem 3.1.

Corollary 3.2 Let $K$ be a nonempty closed convex subset of a real Hilbert space $H$. Let $A$ be an $\alpha$-inverse strongly monotone operator of $K$ into $H$ and let $B$ be a maximal monotone operator on $H$ such that the domain of $B$ is contained in $K$. Let $T: K \rightarrow K$ be a quasinonexpansive mapping such that $I-T$ is demiclosed at zero. Assume that $\operatorname{Fix}(T) \cap(A+$ $B)^{-1} 0 \neq \emptyset$. Let $\left\{\alpha_{n}\right\}$ and $\left\{\beta_{n}\right\}$ be sequences in $(0,1)$ and let $\left\{u_{n}\right\}$ be a sequence in $K$. Let $\left\{x_{n}\right\}$ be a sequence generated by

$$
\left\{\begin{array}{l}
x_{1} \in K \quad \text { chosen arbitrarily, } \\
y_{n}=\alpha_{n} u_{n}+\left(1-\alpha_{n}\right) J_{\lambda_{n} B}\left(x_{n}-\lambda_{n} A x_{n}\right), \\
x_{n+1}=\beta_{n} x_{n}+\left(1-\beta_{n}\right) T y_{n} .
\end{array}\right.
$$

If conditions (c1)-(c4) are satisfied, then the sequence $\left\{x_{n}\right\}$ converges strongly to the element $P_{\mathrm{Fix}(T) \cap(A+B)^{-1} 0} \mathcal{u}$. 
Proof Letting $C=0$ and $D=\partial \delta_{K}$ in Theorem 3.1, the desired result follows.

Let us consider the variational inequality problem. Recall that the subdifferential $\partial \delta_{K}$ of $\delta_{K}$ is a maximal monotone operator and $V I(K, A)=\left(A+\partial \delta_{K}\right)^{-1} 0$, where $A$ is an inverse strongly monotone mapping. We obtain the following result.

Corollary 3.3 Let $K$ be a nonempty closed convex subset of a real Hilbert space H. Let $A$ be an $\alpha$-inverse strongly monotone operator of $K$ into $H$ and let $T: K \rightarrow K$ be a quasi-nonexpansive mapping such that $I-T$ is demiclosed at zero. Assume that $\operatorname{Fix}(T) \cap$ $V I(K, A) \neq \emptyset$. Let $\left\{\alpha_{n}\right\}$ and $\left\{\beta_{n}\right\}$ be sequences in $(0,1)$ and let $\left\{u_{n}\right\}$ be a sequence in $K$. Let $\left\{x_{n}\right\}$ be a sequence generated by

$$
\left\{\begin{array}{l}
x_{1} \in K \quad \text { chosen arbitrarily, } \\
y_{n}=\alpha_{n} u_{n}+\left(1-\alpha_{n}\right) P_{K}\left(x_{n}-\lambda_{n} A x_{n}\right), \\
x_{n+1}=\beta_{n} x_{n}+\left(1-\beta_{n}\right) T y_{n} .
\end{array}\right.
$$

If conditions (c1)-(c4) are satisfied, then the sequence $\left\{x_{n}\right\}$ converges strongly to the element $P_{\mathrm{Fix}(T) \cap V I(K, A)} u$.

Proof Corollary 3.2 easily yields the desired result.

Remark Corollaries 3.2 and 3.3 improve and extend Theorem 3.1 of Takahashi et al. [22] and Theorem 4.2 of Takahashi and Takahashi [23] in the following aspects, respectively.

(1) The nonexpansive mapping is extended to the quasi-nonexpansive mapping.

(2) The constant vector $u$ is replaced by the variables $u_{n}$ with $\lim _{n \rightarrow \infty} u_{n}=u$.

(3) The condition $\lim _{n \rightarrow \infty}\left(\lambda_{n+1}-\lambda_{n}\right)=0$ is removed.

\section{Competing interests}

The authors declare that they have no competing interests.

Authors' contributions

All authors read and approved the final manuscript.

\section{Acknowledgements}

The authors are grateful to referees and editors for their valuable comments and suggestions.

Received: 11 February 2014 Accepted: 30 July 2014 Published: 21 Aug 2014

\section{References}

1. Cai, Y, Tang, Y, Liu, L: Iterative algorithms for minimum-norm fixed point of nonexpansive mapping in Hilbert space. Fixed Point Theory Appl. 2012, 49 (2012)

2. Cui, YL, Liu, X: Notes on Browder's and Halpern's methods for nonexpansive mappings. Fixed Point Theory 10, 89-98 (2009)

3. Halpern, B: Fixed points of nonexpanding maps. Bull. Am. Math. Soc. 73, 957-961 (1967)

4. Mann, WR: Mean value methods in iteration. Proc. Am. Math. Soc. 4, 506-510 (1953)

5. Wittmann, R: Approximation of fixed points of nonexpansive mappings. Arch. Math. 58, $486-491$ (1992)

6. Itoh, S, Takahashi, W: The common fixed point theory of single-valued mappings and multivalued mappings. Pac J. Math. 79, 493-508 (1978)

7. Kocourek, P, Takahashi, W, Yao, JC: Fixed point theorems and weak convergence theorems for generalized hybrid mappings in Hilbert spaces. Taiwan. J. Math. 14, 2497-2511 (2010)

8. Combettes, PL, Pesquet, JC: Image restoration subject to a total variation constraint. IEEE Trans. Image Process. 13 1213-1222 (2004)

9. Kim, GE: Weak and strong convergence theorems of quasi-nonexpansive mappings in a Hilbert space. J. Optim Theory Appl. 152, 727-738 (2012) 
10. Yamada, I, Ogura, N: Hybrid steepest descent method for variational inequality problem over the fixed point set of certain quasi-nonexpansive mappings. Numer. Funct. Anal. Optim. 25, 619-655 (2004)

11. Chuang, CS, Lin, L, Takahashi, W: Halpern's type iterations with perturbations in Hilbert spaces: equilibrium solutions and fixed points. J. Glob. Optim. 56, 1591-1601 (2013)

12. Lin, LJ, Chuang, CS, Yu, ZT: Fixed point theorems for some new nonlinear mappings in Hilbert spaces. Fixed Point Theory Appl. 2011, 51 (2011)

13. Marino, G, Xu, HK: Weak and strong convergence theorems for strict pseudo-contractions in Hilbert spaces. J. Math. Anal. Appl. 329, 336-346 (2007)

14. Martinet, B: Regularisation dinequations variationnelles par approximations successives. Rev. Fr. Inf. Rech. Oper. Ser. 4 154-158 (1970)

15. Rockafellar, RT: Monotone operators and proximal point algorithm. SIAM J. Control Optim. 14, $877-898$ (1976)

16. Güler, O: On the convergence of the proximal point algorithm for convex minimization. SIAM J. Control Optim. 29, 403-419 (1991)

17. Kamimura, S, Takahashi, W: Approximating solutions of maximal monotone operators in Hilbert spaces. J. Approx. Theory 106, 226-240 (2000)

18. Xu, HK: A regularization method for the proximal point algorithm. J. Glob. Optim. 36, 115-125 (2006)

19. Aoyama, K, Kimura, Y, Takahashi, W, Toyoda, M: On a strongly nonexpansive sequence in Hilbert spaces. J. Nonlinear Convex Anal. 8, 471-489 (2007)

20. Takahashi, W, Wong, NC, Yao, JC: Two generalized strong convergence theorems of Halpern's type in Hilbert spaces and applications. Taiwan. J. Math. 16, 1151-1172 (2012)

21. Lin, LJ, Takahashi, W: A general iterative method for hierarchical variational inequality problems in Hilbert spaces and applications. Positivity 16, 429-453 (2012)

22. Takahashi, S, Takahashi, W, Toyoda, M: Strong convergence theorems for maximal monotone operators with nonlinear mappings in Hilbert spaces. J. Optim. Theory Appl. 147, 27-41 (2010)

23. Takahashi, S, Takahashi, W: Strong convergence theorem for a generalized equilibrium problem and a nonexpansive mapping in a Hilbert space. Nonlinear Anal. 69, 1025-1033 (2008)

24. Rockafellar, RT: On the maximal monotonicity of subdifferential mappings. Pac. J. Math. 33, 209-216 (1970)

25. Chang, SS, Lee, HWJ, Chan, CK: Generalized system for relaxed cocoercive variational inequalities in Hilbert spaces. Appl. Math. Lett. 20, 329-334 (2007)

26. Chang, SS, Lee, HWJ, Chan, CK, Liu, JA: A new method for solving a system of generalized nonlinear variational inequalities in Banach spaces. Appl. Math. Comput. 217, 6830-6837 (2011)

27. Wangkeeree, R, Kamraksa, U: A general iterative method for solving the variational inequality problem and fixed point problem of an infinite family of nonexpansive mappings in Hilbert spaces. Fixed Point Theory Appl. 2009, $369215(2009)$

28. Yao, YH, Liou, YC, Yao, JC: An extragradient method for fixed point problems and variational inequality problems. J. Inequal. Appl. 2007, 38752 (2007)

29. liduka, H, Takahashi, W: Strong convergence theorems for nonexpansive mappings and inverse-strongly monotone mappings. Nonlinear Anal. 61, 341-350 (2005)

30. Mainge, PE: Strong convergence of projected subgradient methods for nonsmooth and nonstrictly convex minimization. Set-Valued Anal. 16, 899-912 (2008)

31. Xu, HK: Another control condition in an iterative method for nonexpansive mappings. Bull. Aust. Math. Soc. 65 109-113 (2002)

10.1186/1029-242X-2014-318

Cite this article as: Wu et al.: Strong convergence theorems for quasi-nonexpansive mappings and maximal monotone operators in Hilbert spaces. Journal of Inequalities and Applications 2014, 2014:318

\section{Submit your manuscript to a SpringerOpen ${ }^{\ominus}$ journal and benefit from:}

- Convenient online submission

Rigorous peer review

- Immediate publication on acceptance

Open access: articles freely available online

- High visibility within the field

- Retaining the copyright to your article

Submit your next manuscript at $>$ springeropen.com 